\title{
Computerized or Automated Object Recognition and Analysis of Pattern Matching in Runways Using Surface Track Data
}

\author{
JAYANTH DWIJESH H P \\ Assistant Professor,Dept of ECE, \\ BGS Institute of Technology, \\ ACU Campus, BG Nagara, \\ NgamangalaTQ, Mandya District, \\ India-571448 \\ Email:- dwijeshjayanth@gmail.com
}

\author{
SANDEEP S V \\ Assistant Professor, Dept of ECE, \\ BGS Institute of Technology, \\ ACU Campus, BG Nagara, \\ Ngamangala TQ, Mandya District, \\ India-571448 \\ Email:- sandeepsv79@gmail.com
}

\author{
RASHMI S \\ Assistant Professor, Dept of ECE, \\ BGS Institute of Technology, \\ ACU Campus, BG Nagara, \\ Ngamangala TQ, Mandya District, \\ India-571448 \\ Email:- rashmiarjun27@gmail.com
}

\begin{abstract}
In today's world, accurate and fast information is vital for safe aircraft landings. The purpose of an EMAS (Engineered Materials Arresting System) is to prevent an aeroplane from overrunning with no human injury and minimal damage to the aircraft. Although various algorithms for object detection analysis have been developed, only a few researchers have examined image analysis as a landing assist. Image intensity edges are employed in one system to detect the sides of a runway in an image sequence, allowing the runway's 3-dimensional position and orientation to be approximated. A fuzzy network system is used to improve object detection and extraction from aerial images. In another system, multi-scale, multiplatform imagery is used to combine physiologically and geometrically inspired algorithms for recognizing objects from hyper spectral and/or multispectral (HS/MS) imagery. However, the similarity in the top view of runways, buildings, highways, and other objects is a disadvantage of these methods. We propose a new method for detecting and tracking the runway based on pattern matching and texture analysis of digital images captured by aircraft cameras. Edge detection techniques are used to recognize runways from aerial images. The edge detection algorithms employed in this paper are the Hough Transform, Canny Filter, and Sobel Filter algorithms, which result in efficient detection.
\end{abstract}

Keywords - Remote Sensing, Runway, Hough Transform, Aerial Image, Canny Filter, Sobel Filter, Feature Extraction,

\section{INTRODUCTION}

The number of remote-sensing sensors has been increased at a remarkable rate over the last decades. Observations obtained from a single sensor do not provide required information, so a fusion of remote sensing data with observations from multiple sensors is used to determine the accurate data. Examples include detection of roads and buildings, runway detection as well as other application areas. However, precise determination of the detailed information such as shape and spectral attributes are difficult to derive. In order to provide a safe separation of aircraft on the airport surface, air traffic controllers issue verbal clearances to pilots for sequence of aircraft arrivals, departures, and runway crossings.

Most of the time, the controllers and pilots work together successfully. But at times mistakes do occasionally happen, resulting in hundreds of runway incursions a year and, less frequently, near misses and collisions. With this rate of incursions, it is advantageous to have an independent communication system as a backup to the current system. Runway status lights are installed at runway-taxiway intersections and at departure points along the runways which provides a clear signal to pilots crossing or departing from a runway and warning them of potential conflicts with traffic already on the runway. But still crashes may happen due to similarities in detected object's top view i.e., some buildings top view will resemble that of a runway. This may lead to misinterpretation of data and leads to crash. To overcome these problems a pattern matching technique is added up to the available standard technology. In runway analysis technique, first the aerial images are taken, from the images the objects are detected using edge detection algorithms. In this, the edge detection techniques used are Hough Transform, Canny Filter and Sobel Filter algorithms which lead to efficient detection of runways from aerial images. Then a comparative study is done using the feature extraction technique in which the different features of the object are retrieved and it is compared with the standard values stored in the geospatial database. The output obtained from this is taken for pattern matching test. If the object satisfies both edge detection and pattern matching to the standard features of a runway then the detected object is a runway.

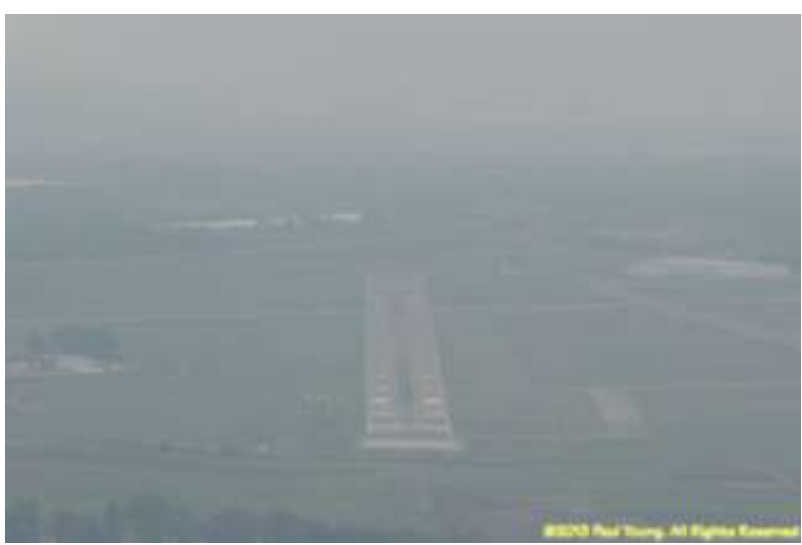

Fig 1: Aerial image of Runway during bad weather

In various fields there is a need to manage geometric, geographic, or spatial data, which means data related to space. It has therefore been suggested to clearly distinguish two classes of systems called spatial database systems and image database systems, respectively. Image database systems may include analysis techniques to extract objects in space from images, and offer some 
spatial database functionality, but are also prepared to store, manipulate and retrieve raster images as discrete entities.

Accurate and timely information is critical for the safe landing of aircraft in now-a-days. The goal of an EMAS (Engineered Materials Arresting System) is to avoid aircraft overrun with no human injury and minimal aircraft damage. Although many techniques have been developed for the analysis of object detection, relatively few researchers have considered image analysis as an aid to aircraft landing. In one system, image intensity edges are used to detect the sides of a runway in an image sequence, and the 3-dimensional position and orientation of the runway can be estimated. For better detection and extraction of objects from an aerial image, a fuzzy network system is used. In another system integration of biologically and geometrically inspired approaches for detecting objects from hyper spectral and/or multispectral (HS/MS), multi-scale, multiplatform imagery is used. But the drawback of these technologies is similarity in the top view of runways and building and roads or other objects. We propose a new method to detect and track the runway using pattern matching and texture analysis using digital images from cameras mounted on the aircraft.

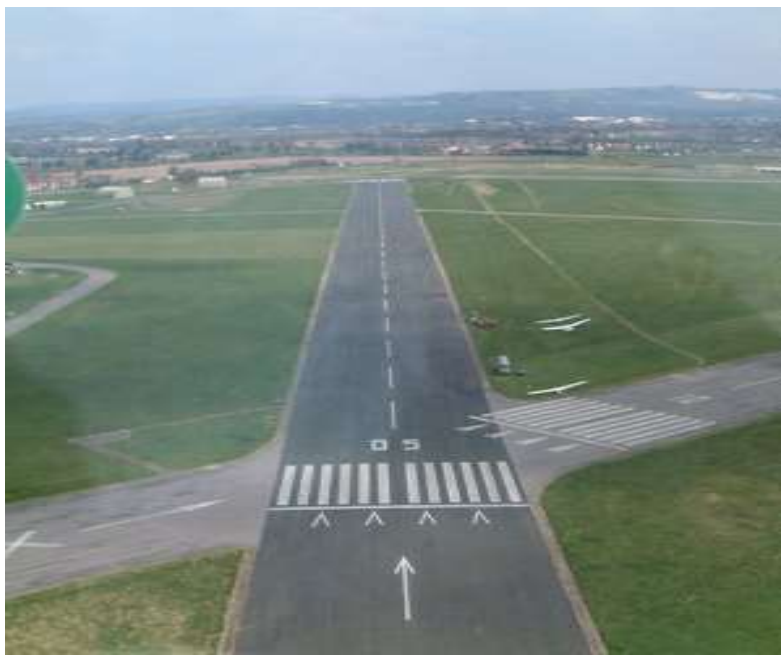

Fig 2: Aerial image of Runway.

In order to detect runway from aerial image edge detection algorithms are used. Two runways pointing in the same direction are classified as dual or parallel runways based on the separation distance. In some countries, it is mandatory in flight rules that only one runway may be used at a time under certain weather conditions even if the parallel runways are too close to each other.

\section{PATTERN MATCHING ANALYSIS}

\section{A. Defination}

Pattern matching is comparing two patterns in order to determine whether they match (i.e., that they are the same) or do not match (i.e., that they differ). Pattern matching is the core procedure of theory-testing with cases. Testing consists of matching an "observed pattern" (a pattern of measured values) with an "expected pattern" (a hypothesis), and deciding whether these patterns match (resulting in a confirmation of the hypothesis) or do not match (resulting in a disconfirmation). Essential to pattern matching (as opposed to pattern recognition, which is a procedure by which theory is built) is that the expected pattern is precisely specified before the matching takes place.

\section{B. The Concept of Pattern Matching}

A pattern is any arrangement of objects or entities. The term "arrangement" indicates that a pattern is nonrandom. Theories "predict" some pattern of values of variables. Such predictions are usually called hypotheses. The term "expected pattern" will be used here for specifications of the hypothesis that allow for a rigorous comparison with an "observed pattern" of values of variables in a test.

Donald T. Campbell coined the term "pattern identification" as a characteristic of qualitative analysis which he defines as holistic (i.e., analyzing the pattern) rather than atomistic (i.e., analyzing its constituents). He argued that the single case study design could provide for a strong test of a theory if an entire set of expectations deduced from that theory (which together would constitute an "expected pattern") could be shown to be true in that case. Campbell also called this a "configurationally approach". He insisted that qualitative analysis in this design tends to disconfirm rather than confirm prior belief due to the requirement that, in the test, each separate element of a pattern or configuration that is observed is exactly as expected. As noted by Thomas D. Cook and Donald T. Campbell, the strength of this "non-equivalent, dependent variables design" is precisely that the variables that constitute the pattern or configuration are non-equivalent, i.e., not substitutable.

\section{Applications}

\section{- Independent Variable Designs}

Campbell's and Yin's approach to pattern matching is implicitly limited to the testing of propositions about characteristics of single cases (which can be tested in single cases) and not about differences between cases (see the entry on Theory-testing with cases). Expected and observed patterns, therefore, consist of values of variables that all pertain to the single case. The simplest type of an independent variable pattern consists of the expected value of only one independent variable (rather than of a number of variables), given the value of a dependent variable. There are only two propositions on which such single point expected patterns can be based, (a) necessary condition propositions, and (b) sufficient condition propositions.

\section{- Necessary condition propositions}

State that an outcome $\mathrm{Y}$ is only possible if condition $\mathrm{X}$ is present. To test such a proposition, a case must be selected in which outcome $\mathrm{Y}$ is present. The expected pattern is: $\mathrm{X}$ is present. (Note that the proposition does not entail any prediction about conditions of the absence of 
Y.) It is observed whether $\mathrm{X}$ is present in the selected case. The observed pattern is either that $\mathrm{X}$ is present or that $\mathrm{X}$ is absent. Pattern matching in this case consists of checking what the value of $\mathrm{X}$ is in the observed pattern.

\section{- Sufficient condition propositions:}

State that an outcome $\mathrm{Y}$ is always present when condition $\mathrm{X}$ is present. To test such a proposition, a case must be selected in which outcome $\mathrm{Y}$ is absent. (Note that the proposition does not entail any prediction about conditions of the presence of Y.) The expected pattern is: $\mathrm{X}$ is absent. Pattern matching in this case consists of checking what the value of $\mathrm{X}$ is in the observed pattern.

\section{- Configurationally theories}

Usually specify a number of conditions that together, i.e. in a configuration, must be present for an outcome to exist. If a configuration consists of, say, four elements, these can be seen as four separate necessary conditions. Four single point independent variable patterns could be specified and tested as described above for the necessary condition proposition, but it is also possible to specify a single four-point pattern (e.g., $[\mathrm{A}+/ \mathrm{B}+/ \mathrm{C}+/ \mathrm{D}+]$ ) which is expected to be observed in a case in which the outcome is present. The observed and the expected pattern do not match if any of the four variables is absent and, in such a case, the hypothesis is disconfirmed.

\section{- Process theories}

Are a types of configurationally theory in which not only the presence of a number of conditions is specified but also their temporal order. The expected pattern in a case in which $\mathrm{Y}$ is present is, for instance, $[\mathrm{A}+\square \mathrm{B}+\square$ $\mathrm{C}+\square \mathrm{D}+]$. The observed pattern must reflect the temporal order in which conditions A, B, C, and D occurred (if at all) and a match is confirmed only A, B, C, and D have the same temporal place in expected and the observed pattern.

\section{RUNWAY SPECIFICATION}

\section{A. specific features}

There are some specific features of a runway which are listed below.

1) Runways have markings and signs on it. In the case of larger runways a distance remaining sign Representation (black box with white numbers) is used. A single number is used to indicate thousands of feet remaining. For example '7' will indicate 7,000 ft $(2,134 \mathrm{~m})$ ' remaining.

2) A naming system is followed for runways in which it is assigned a number between 01 and 36, which is one Tenth of the magnetic azimuth of the runway's heading.

For example, a runway numbered '09'points east $\left(90^{\circ}\right)$, a Runway numbered ' $27^{\prime}$ points west $\left(270^{\circ}\right)$, a runway numbered ' $36^{\prime}$ points north $\left(360^{\circ}\right.$ rather than $\left.0^{\circ}\right)$ and a Runway numbered ' $18^{\prime}$ points south $\left(180^{\circ}\right)$.
3) The dimensions of the runway varies from $245 \mathrm{~m}$ (804

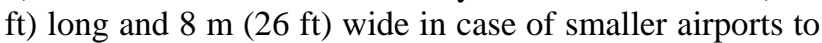
$5,500 \mathrm{~m}(18,045 \mathrm{ft})$ long and $80 \mathrm{~m}(262 \mathrm{ft})$ wide in case of large international airports to the huge 11,917 $\mathrm{m}(39,098$ $\mathrm{ft})$ x $274 \mathrm{~m}(899 \mathrm{ft})$ lake bed runway

$17 / 35$.

4) The minimum distance between the border lines of the runway is $75 \mathrm{ft}(23 \mathrm{~m})$, and the maximum is Approximately $200 \mathrm{ft}(61 \mathrm{~m})$. The maximum distance Between the lights for detecting the runway within each Line is $200 \mathrm{ft}(61 \mathrm{~m})$. The minimum length of the two Borders of the runway are 1,400 $\mathrm{ft}(427 \mathrm{~m})$. There should be a minimum of 8 lights in the line. Two runways pointing in the same direction are classified as dual or parallel runways based on the separation Distance. In some countries, it is mandatory in flight rules that only one runway may be used at a time under certain Weather conditions even if the parallel runways are too close to each other. The structure of runway is as shown in the below figure(3)

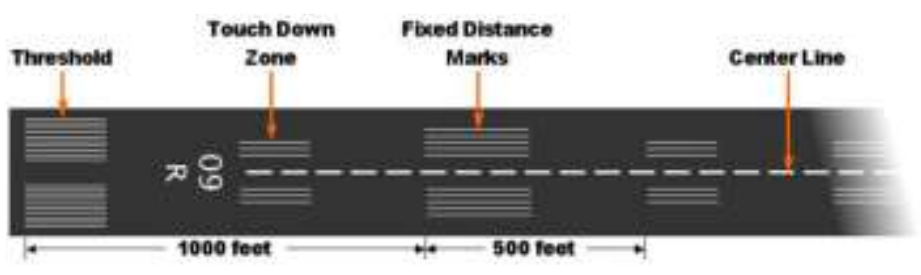

Figure 3: Structure of Runway.

\section{B. Objectives}

The objective of this work is to detect the runway automatically (unmanned) without consuming huge amount of time in order to avoid disasters. To meet this objective enhancements are done on each of the following algorithms

- Hough transform

- Canny Filter

- Sobel Filter

- Blob detection

\section{THE PROPOSED WORK}

The algorithm consists of the initial runway edge detection and the follow up tracking of road borders. The initial edge detection uses the Hough transform in the path tracking Random Hough Transform on the Region of Interest to improve computing speed.

There are some problems of canny operator, unable to detect the weak edge and distinguish the grayscale with little change, the detected edge un continuous. Based on these defects, the Mallat wavelet transform us used to reinforce the weak edge of input images, quadratic optimization of genetic algorithm to get a proper threshold in self adapting standard during canny algorithm steps. With the base of canny operator and the improvement, here we build a new model, which satisfies the need of pavement edge detection real time with is a 
less time consuming process. The best known is Canny edge detection that combine a Gaussian low pass filter for noise reduction and non-maximal suppression and hysteresis threshold for edge localization.

A possible problem of this approach is that the threshold values. Applying a single fixed threshold to gradient maxima is not an optimal choice. Thus, Canny uses two thresholds values namely $\mathrm{T}_{\text {low }}$ and $\mathrm{T}_{\text {high }}$ to reduce the number of false positive of pixels that represent significant contours in the image. However, by introducing two fixed threshold values are also not an optimal choice due to high variations in images. A method that computes the threshold values from the foreground and background image pixels are used. According to this method, an image is divided into several blocks using at multiple resolution levels. After that, a sampling approach is used on global and local regions to get the optimal thresholds by selecting the highest between class variance values.

Thus it provides an advanced algorithm based on classical Sobel operator whose defect is rough effect for edge detection and extremely sensitive to the noise. Here we propose an improved algorithm which used the improved Sobel operator with a combination of median filtering method. This algorithm makes the edge detection for the Salt and pepper noise images and it effectively overcome the problem that the Sobel operator is only sensitive to vertical and horizontal direction. It combines the advantage of the median filter to remove salt-pepper noise .

The detection of runway is carried out by using the algorithms or methods. The general structure of runway detection approach is done as in Fig (4). In Feature extraction technique the feature of the object detected is compared with the standard details in the geo spatial database. If every feature of the object is matching with the standard features of the runway, the object is confirmed as runway. Fig (5) represents the feature extraction phase.

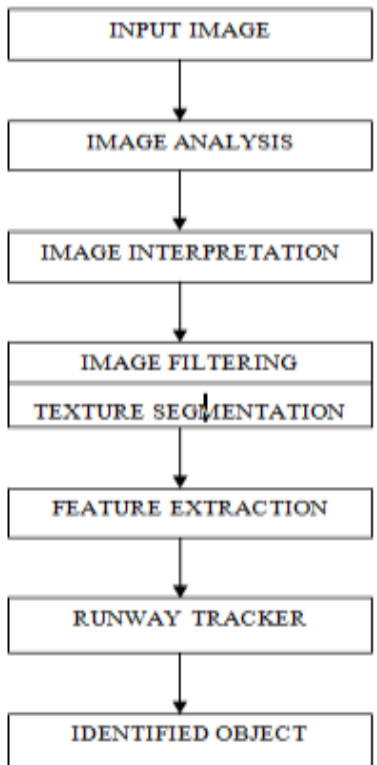

Fig 4: Runway detection approach
The aerial image is taken with the help of a camera and the original image obtained is passed on to the next stage i.e., Pre Processing Phase. In the image preprocessing phase, the original image is converted to grayscale image and then the corresponding binary details of the image is obtained .From this binary information the edge detection algorithm is applied to detect the edges of the object and thus the output transformed image is obtained. The edge detection algorithm used includes Hough Transform and Canny Filter in which Canny Filter provides a better edge detection technique.

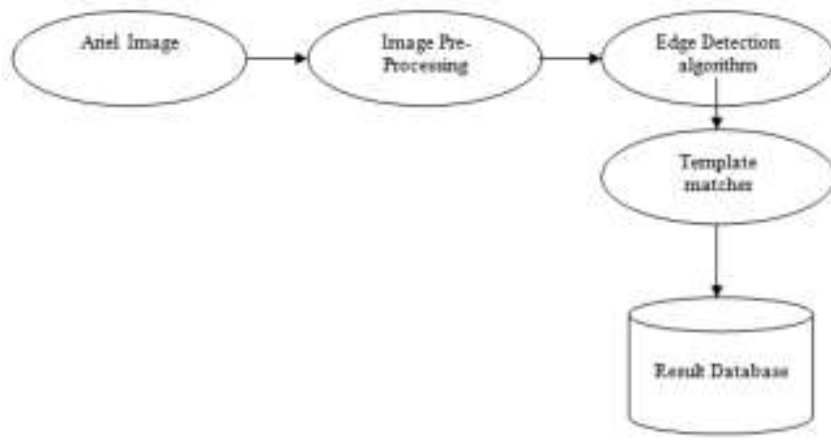

Fig 5: Feature Extraction

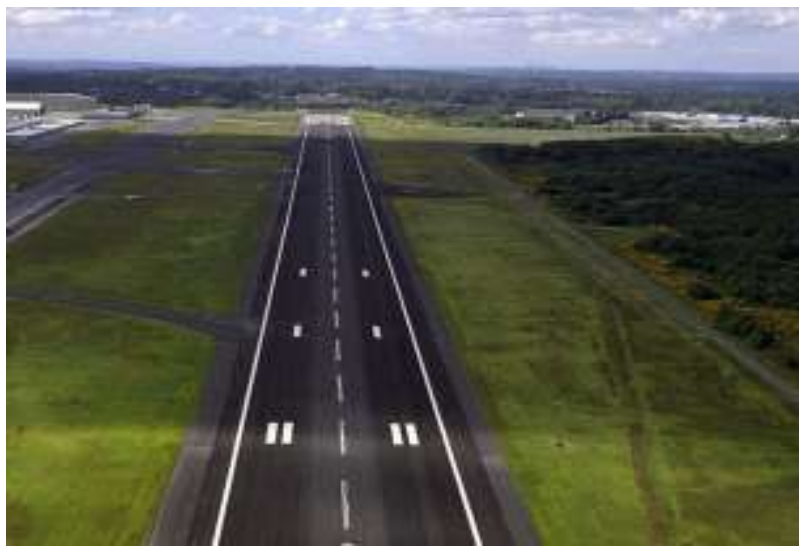

Fig 6: Input aerial image of a runway during normal weather condition using canny filter

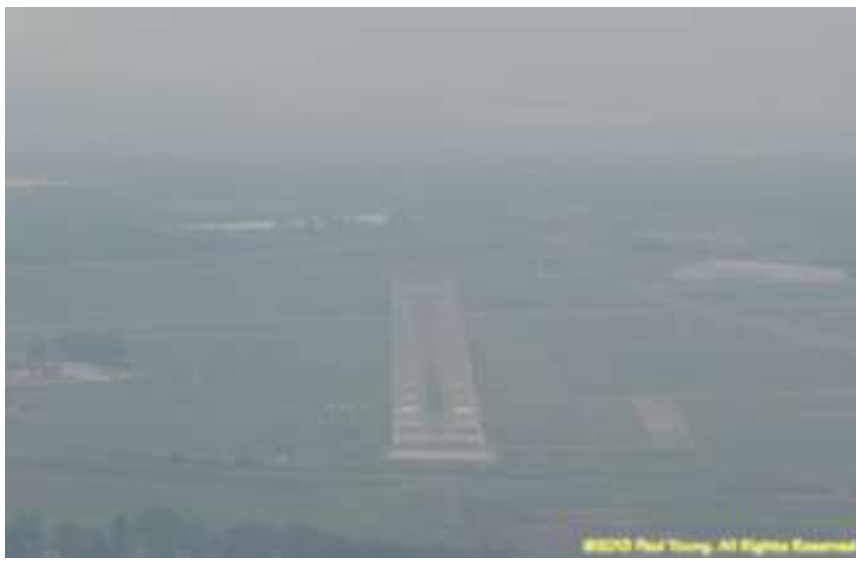

Fig 7: Input aerial image of a runway during bad weather condition using Sobel filter 
For the texture based method color comparison in image pixels are compared individually with a predetermined color vector to identify possible points on the runway. It includes the lighting of runways on a rainy day or a foggy day. In Feature extraction technique the feature of the object detected is compared with the standard details in the geo-spatial database. If every feature of the object is matching with the standard features of the runway, the object is confirmed as runway. In order to validate the algorithm we make use of a serial of aerial images and image processing is performed for detection of runway edges. Image Preprocessing is carried out by blob detection and template matcher techniques and the results are stored in the results database. The comparison between the previous and present work is done.

\section{EXPERMINATAL RTESULTS AND ANALYSIS}

\section{A. Sobel filter}

- Aim is to compute an approximation of the gradient of the image intensity function.

- The Sobel operator is based on convolving the image with a small, separable, and integer valued filter in horizontal and vertical direction.

- Uses two $3 \times 3$ kernels which are applied separately and independently

- Its convolved with the original image to calculate approximations of the derivatives one for horizontal changes, and one for vertical.

\begin{tabular}{|l|l|l|}
\hline-1 & 0 & +1 \\
\hline-2 & 0 & +2 \\
\hline-1 & 0 & +1 \\
\hline
\end{tabular}

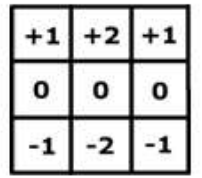

Fig 8: Sobel operators

- If we define $\mathbf{A}$ as the source image, and $\mathbf{G}_{\mathrm{x}}$ and $\mathbf{G}_{\mathrm{y}}$ are two images which at each point contain the horizontal and vertical derivative approximations, then computations is

$\mathbf{G}_{x}=\left[\begin{array}{rrr}+1 & 0 & -1 \\ +2 & 0 & -2 \\ +1 & 0 & -1\end{array}\right] * \mathbf{A}$ and $\mathbf{G}_{y}=\left[\begin{array}{rrr}+1 & +2 & +1 \\ 0 & 0 & 0 \\ -1 & -2 & -1\end{array}\right] * \mathbf{A}$

- Where *here denotes the 2dimensional convolution operation.

- The x-coordinate is defined as increasing in the "right"-direction, and the y-coordinate is defined as increasing in the "down"-direction.

- At each point in the image, the resulting gradient approximations can be combined to give the gradient magnitude, using:

$$
\mathrm{G}=\sqrt{ } \mathrm{G}_{\mathrm{x}}^{2}+\mathrm{G}_{\mathrm{y}}^{2}
$$

- Using this information, we can also calculate the gradient's direction:

$$
\theta=\arctan \left(\frac{\left|G_{\mathrm{y}}\right|}{\left|G_{\mathrm{x}}\right|}\right)
$$

Where, for example, $\boldsymbol{\theta}$ is 0 for a vertical edge which is darker on the right side.

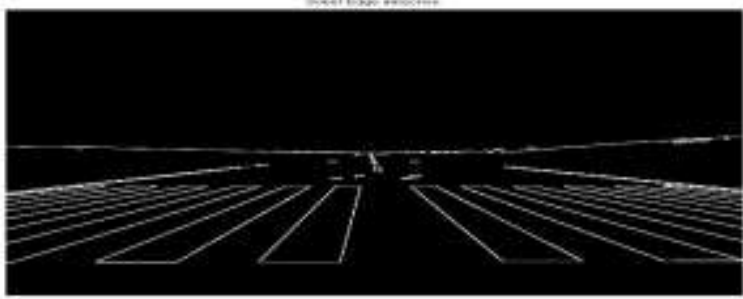

Fig 9: Sobel Edge detection

- Steps involved in sobel edge detection:-

Input: A Sample Image.

Step 1: Accept the input image.

Step 2: Apply mask Gx, Gy to the input image.

Step 3: Apply Sobel edge detection algorithm and the gradient.

Step 4: Masks manipulation of Gx, Gy separately on the input image.

Step 5: Results combined to find the absolute magnitude of the gradient.

Step 6: The absolute magnitude is the output edges.

Output: Detected Edges.

\section{B. Canny filter}

- The Canny edge detector is an edge detection operator that uses a multi-stage algorithm to detect a wide range of edges in images.

- Its aim is to discover the optimal edge detection algorithm which means good detection ,good localization ,Number of response.

- $\quad$ stages of the canny algorithm

Stage 1: Noise reduction

Stage 2: Finding the intensity gradient of the image

Stage 3: Non-maximum suppression

Stage 4: Double thresholding

Stage 5: Edge tracking by hysteresis

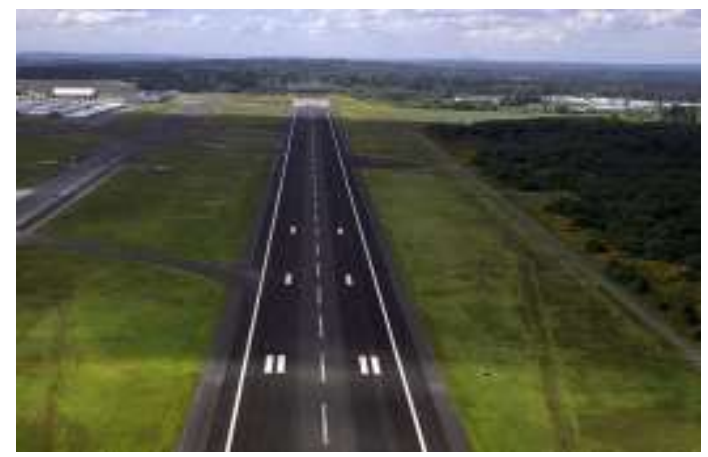

Fig (a) 


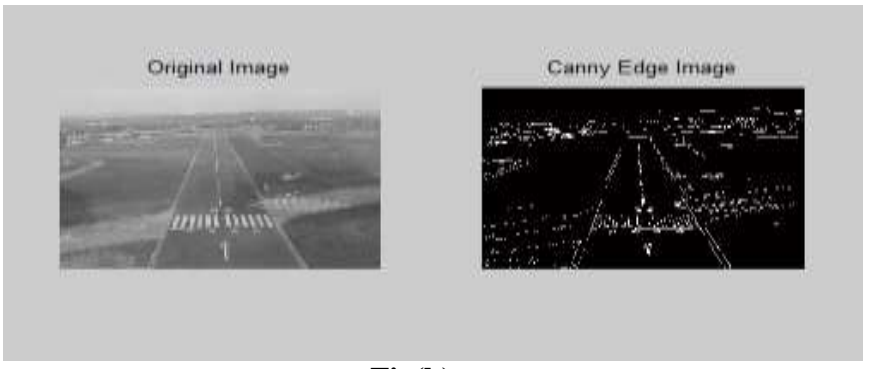

Fig(b)

Fig $10(a \& b)$ : Snapshot shows the result obtained during implementation of Canny filter.

\section{Hug transform}

- The Hough transform is a feature extraction technique used in image analysis, computer vision, and digital image processing.

$$
\theta=\arctan \left(\frac{\left|G_{y}\right|}{\left|G_{x}\right|}\right)
$$

- The purpose of the technique is to find imperfect instances of objects within a certain class of shapes by a voting procedure.

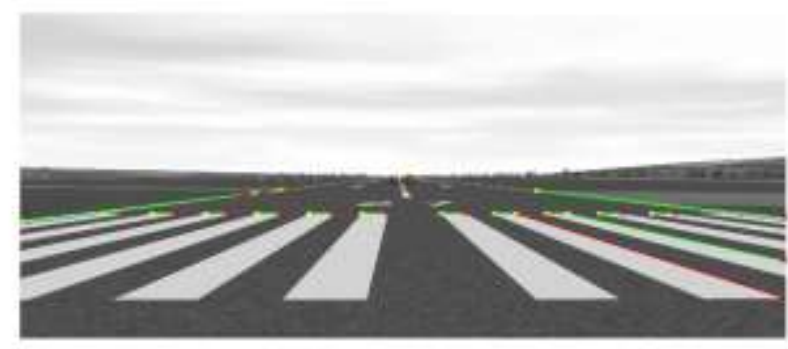

Fig 11: Hough edge detector

- A line can be represented by two numbers

- Here we will represent the blue line by $(w, \theta)$

- In other words we define it using - a line from an agreed origin of length $\mathrm{w}$ at angle $\theta$ to the horizontal

- Each point if considered as isolation could lie on an infinite number of straight lines.

- In the Hough transform each point votes for every line it could be on

- The lines with the most votes win

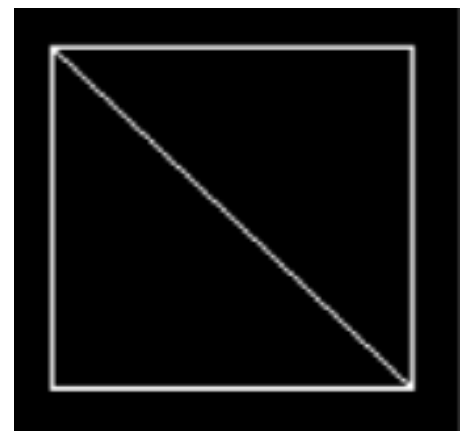

Fig12: Hough space

\section{Blob filter}

- Blob detection refers to mathematical methods that are aimed at detecting regions in a digital image that differ in properties, such as brightness or color, compared to areas surrounding those regions.

- Two main classes of blob detectors:

(i) Differential methods are based on derivatives of the function with respect to position.

(ii) Methods based on local extrema are based on finding the local maxima and minima of the function.

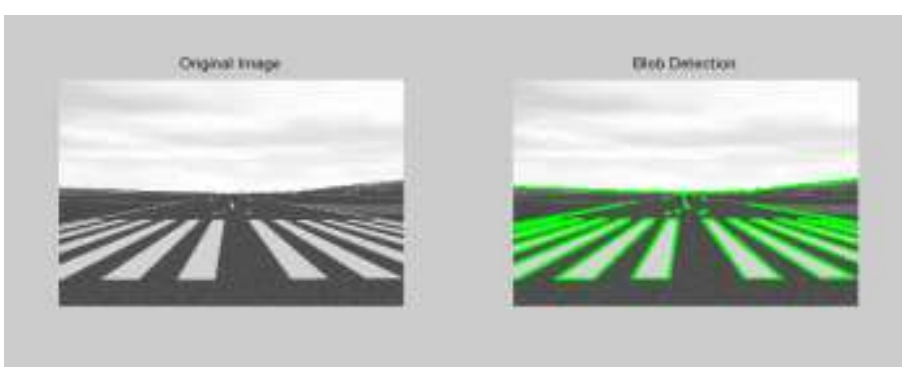

Fig 13: snapshot shows the result obtained during the implementation of blob filter

\section{CONCLUSION}

In this research, we offer a method for automatically detecting runways and their characteristics. This is an expansion of the runway detection technology that employs pattern recognition. Experiments with several aerial photos were undertaken, and an accurate transformed image was obtained. The intrinsic characteristics of the various runways are highlighted in this paper. The input for this system was aerial photos, which were processed using object recognition, feature extraction, and pattern recognition techniques. The result of our effort generates a runway detecting system which is efficient than the existing strategies.

\section{REFERENCES}

[1] ChunxiMa, WenshuoGao; Lei Yang; Zhonghui Liu, "An improved Sobel algorithm based on median filter", Page(s): V1-88 - V1-92, ISBN: 978-1-42447479-0, 1-3 Aug. 2010, IEEE.

[2] HuiliZhao, Guofeng Qin; Xingjian Wang, "Improvement of canny algorithm basedon pavement edge detection", Page(s): 964 - 967, ISBN: 978-14244-6513-2, 16-18 Oct. 2010, IEEE.

[3] WenshuoGao, Xiaoguang Zhang; Lei Yang; Huizhong Liu, "An improved Sobel edge detection", Page(s): 67 - 71, ISBN: 978-1-4244-5537-9, 9-11 July 2010, IEEE.

[4] Chen Fang-fang, Jiang Xing-fang; Jiang Zhong-yi, "The Application of Improved Canny Algorithm in Internal Waves' Parameters from MODIS Remote Sensing Images", Page(s): 1 - 3, ISBN: 978-1-42444963-7, 19-21 June 2010, IEEE. 
[5] JianfengWang, Ye Wu; Zehua Liang; YuanjunXi,"Lane detection based on random Hough transform on region of interesting", Page(s): 1735 1740, ISBN: 978-1-4244-5701-4, 20-23 June 2010, IEEE.

[6] Phueakjeen, W., Jindapetch, N. ; Kuburat, L. ; Suvanvorn, N., "A study of the edge detection for road lane", Page(s): 995 - 998, ISBN: 978-1-4577-0425-3, 17-19 May 2011,IEEE.

[7] ZhongZhang, Geng Zhao, "Butterworth filter and Sobel edge detection to image", Page(s): 254 - 256, ISBN: 978-1-61284-771-9, 26-28 July 2011, IEEE.

[8] XiaobingWang, Baokui Li; QingboGeng, "Runway Detection and Tracking for Unmanned Aerial Vehicle Based on an Improved Canny Edge Detection Algorithm", Page(s): 149 - 152, ISBN: 978-1-46731902-7, 26-27 Aug. 2012, IEEE.

[11] Jose, Abin, Seelamantula, Chandra Sekhar, "Bilateral edge detectors", , Page(s): 1449 - 1453, ISSN : 15206149, 26-31 May 2013,IEEE.

[12] JianfengWang, Ye Wu; Zehua Liang; YuanjunXi,"Lane detection based on random Hough transform on region of interesting", Page(s): 1735 1740, ISBN: 978-1-4244-5701-4, 20-23 June 2010, IEEE.

[13] HuiliZhao, Guofeng Qin; Xingjian Wang, "Improvement of canny algorithm based on pavement edge detection", Page(s): 964 - 967, ISBN: 978-1-4244-6513-2, 16-18 Oct. 2010, IEEE.

[14] ChunxiMa, WenshuoGao; Lei Yang; Zhonghui Liu, "An improved Sobel algorithm based on median filter", Page(s): V1-88 - V1-92, ISBN: 978-1-42447479-0, 1-3 Aug. 2010, IEEE.

[9] Othman, Z. , Abdullah, A. ; Prabuwono, A.S., "A statistical approach of multiple resolution levels for canny edge detection", Page(s): 837 - 841,ISBN: 978-1-4673-5117-1, 27-29 Nov. 2012,,IEEE.

[10] Wang Xiaoyun, Wang Yongzhong; Wen Chenglin,"Robust lane detection based on gradientpairs constraint", Page(s): 3181 - 3185, ISBN: 978988-17255-9-2, 22-24 July 2011, IEEE.

[15] Nikhil Gupta, Rahul Gupta, Amardeep Singh, Matt Wytock Object Recognition using Template Matching, pp 1-6. December 12, 2008. 